200

\section{SORVEGLIANZA DELL'INFEZIONE /MALATTIA DA TOXOPLASMA GONDII IN PAZIENTI TRAPIANTATI DI ORGANO SOLIDO TORACICO}

Genco F., Meroni V.,Sarchi E.,Castiglioni B., DiMatteo A.,Zerrilli E,.Minoli L.

\section{Dipartimento di Clinica di Malattie Infettive \\ Università degli Studi Pavia \\ IRCCS Policlinico San Matteo Pavia \\ Via Taramelli, 5 27 I00 Pavia}

Introduzione. L'infezione da Toxoplasma gondii è una possibile complicanza nei pazienti trapiantati di organo solido toracico in Europa dove la sieroprevalenza è alta. La toxoplasmosi può essere causata dalla presenza di cisti nel cuore trapiantato in un ricevente sieronegativo oppure dalla riattivazione di una pregressa infezioni di pazienti sieropositivi. Scopo dello studio è stato:

1) valutare la frequenza di siero conversione per toxoplasmosi in riceventi sieronegativi di organo solido (cuore e/o polmone) con donatori sieropositivi (R-/ D+) o sieronegativi (R-/D-).

2) Verificare l'efficacia della chemioprofilassi in R-/D+ e della profilassi igienico alimentare in tutti i riceventi sieronegativi.

3) Valutare le caratteristiche clinico sierologiche della malattia in tutti i pazienti infetti.

Metodi. Abbiamo valutato 114 riceventi sieronegativi sottoposti a follow-up clinico e sierologico dopo il trapianto per diversi intervalli di tempo (2 mesi- 14 anni) presso il Dipartimento di Malattie Infettive. I pazienti erano così suddivisi R-/D-: 50 pazienti follow-up da 2 a 198 mesi, 38 maschi, 36 cuore, 13 polmone, 1 polmone/fegato.

Solo profilassi igienico alimentare.

R-/D+: 64 pazienti follow-up da 2 a 151 mesi, 46 maschi, 51 cuore, 13 polmone. Profilassi igienico alimentare e terapia con PYR+SUL per due mesi. Tutti i pazienti sono stati testati per anticorpi IgG IgM con i seguenti tests: ELISA IgG $\operatorname{IgM}$ ( Diasorin Saluggia Italia), IgG ELFA (Biomerieux Marcy L'Etoile).In tutte le sospette sieroconversioni anche con Toxo IgM ISAGA, Toxo IgG Avidity (Biomerieux Marcy L'Etoile France), IgA Elisa (Diasorin Saluggia Italia), IgG IgM Western-Blot (LDBIO Lyon France). Nei casi sintomatici è stata anche eseguita una nested PCR con gene target AF 487580 (Clonit Milano Italia).

Risultati. Sono mostrati nella seguente tabella

\begin{tabular}{llll}
\hline & $\mathrm{R}-/ \mathrm{D}+$ & $\mathrm{R}-/ \mathrm{D}-$ & Tot \\
\hline Sieroconversioni & $14(22 \%)$ & $5(10 \%)$ & 19 \\
\hline no sieroconversioni & 50 & 45 & 95 \\
\hline Tot & 64 & 50 & 114
\end{tabular}

Conclusioni. Nel nostro studio non abbiamo osservato nessuna differenza statisticamente significativa tra pazienti R-/D- R$/ \mathrm{D}+$ per quanto concerne la frequenza di sieroconversioni, tuttavia la piccola dimensione campionaria riduce l'accuratezza statistica. I nostri dati confermano anche l'efficacia dei due mesi di chemioprofilassi nei pazienti R-/D+.. R-/D- devono seguire profilassi igienico alimentare e ripetere prelievi ogni 6 mesi per verificare al più presto la sieroconversione.Tutti $i$ pazienti R-/D+ devono seguire la profilassi igienico alimentare e fare la chemioprofilassi per almeno due mesi.
201

\section{MONITORAGGIO DELLA PROFILASSI PER LA TUBERCOLOSI LATENTE IN PAZIENTI TRAPIANTATI CON IL TEST T SPOT-TB}

\author{
Arosio M.', Bargiggia G.', Rizzi M.', Nozza F. ', Grigis A.', \\ Goglio A'.
}

USC Microbiologia e Virologia,

${ }^{2}$ Malattie Infettive,

A.O. Ospedali Riuniti di Bergamo,Largo Barozzi I.

Introduzione. Negli ultimi anni sono stati introdotti test che permettono la rilevazione della citochina IFN- $\gamma$ prodotta dai linfociti a seguito della stimolazione con antigeni specifici di M. tuberculosis. Tra questi il test T SPOT-TB (distribuito in Italia da Nanogen) che utilizza gli antigeni ESAT-6 e CFP-10 presenti nella regione RD1 del genoma tubercolare e che dimostra sensibilità e specificità maggiore del test tubercolinico in particolare nei confronti dei pazienti con sistema immunitario compromesso (terapie immunosoppressive, HIV-positivi), nei bambini e negli anziani, soggetti in cui il test cutaneo risulta a volte dubbio o negativo.

Lo scopo del presente lavoro è stato di monitorare l'effetto della profilassi antitubercolare sulla risposta $\mathrm{T}$ cellulare agli antigeni specifici di M. tuberculosis.

Pazienti e metodi. Nel giugno 2005, a seguito del riscontro di un caso di tubercolosi in Gastroenterologia ed uno in Medicina, 22 pazienti afferenti alle USC di Gastroenterologia e Medicina, immunodepressi per patologie varie, sono stati sottoposti a screening con T SPOTTB (T-0). Solo per i negativi, il test veniva ripetuto a distanza di un mese (T-1, due mesi dalla esposizione) e ricontrollati i pazienti positivi dopo un periodo di sei mesi in profilassi con isoniazide (T-2).

Risultati. Dal gruppo indagato, 7 pazienti (range d'età 49 68 aa.) sottoposti a terapia immunosoppressiva per trapianto di fegato $(<6$ mesi fino a 4 anni) sono risultati positivi al $\mathrm{T}$ SPOT-TB. I risultati sono esposti nella tabella seguente.

\begin{tabular}{|c|cc|cc|cc|}
\hline \multirow{2}{*}{ Casi } & \multicolumn{2}{|c|}{ T-O } & \multicolumn{2}{c|}{ T-1 } & \multicolumn{2}{c|}{ T-2 } \\
\cline { 2 - 7 } & \multicolumn{2}{|c|}{ ESAT 6- } & \multicolumn{2}{c|}{ ESAT 6- } & \multicolumn{2}{c|}{ ESAT 6- } \\
& Esito & CFP 10 & Esito & CFP 10 & Esito & CFP 10 \\
\hline GP & POS & $7-9$ & - & - & Neg & $1-2$ \\
DF & Neg & $0-0$ & POS & $5-8$ & Neg & $1-0$ \\
MS & Neg & $2-5$ & POS & $15-15$ & Neg & $3-0$ \\
GF & POS & $3-7$ & - & - & Dubbio & $2-6$ \\
FQ & POS & $25-25$ & - & - & POS & $>50->50$ \\
AM & POS & $2-9$ & - & - & POS & $11-12$ \\
GT & POS & $8-18$ & - & - & POS & $49-27$ \\
\hline
\end{tabular}

Conclusioni. I dati ottenuti confermano quanto già pubblicato in Letteratura: una progressiva riduzione delle cellule $\mathrm{T}$ specifiche che potrebbe correlare con l'efficacia della profilassi/terapia. Nel nostro studio questo fenomeno si osserva nei pazienti con un numero di spot: $\leq 15$ anche se questo criterio non trova conferma in un paziente che continua a rimanere positivo. L'esiguità del campione indagato non consente conclusioni esaustive in merito alla validità del test $\mathrm{T}$ SPOT-TB per il monitoraggio del trattamento della tubercolosi latente e pertanto ulteriori studi sono richiesti. 